\title{
Intervención educativa sobre hipertensión arterial en la tercera edad del Consultorio No 27 de Güines
}

Educational intervention on arterial hypertension in the third age of the Office No. 27 of Güines

Efraín Velasteguí López. ${ }^{1}$ Lic. Joel García Espinosa. ${ }^{2}$ \& Est. Liana Borges Sierra. ${ }^{3}$

\begin{abstract}
.
The subject studied is recreational physical activity, free time and quality of life in the university extension. An educational intervention on arterial hypertension in the elderly was carried out in the Office No. 27 of Güines, Mayabeque province. The research method was used to identify older adults with this pathology and they were applied a routine of physical exercises and dietary recommendations with which patients report feeling more compensated for the disease they have; in this way their own symptoms decrease; using materials such as:
\end{abstract}

\section{Resumen.}

La temática que se estudia es la actividad física recreativa, tiempo libre y calidad de vida en la extensión universitaria. Se realizó una intervención educativa sobre hipertensión arterial en la tercera edad en el Consultorio No 27 de Güines, provincia Mayabeque. Se utilizó el método de pesquisa para identificar los adultos mayores con esta patología y se les aplicó una rutina de ejercicios físicos y recomendaciones dietéticas con los cuales los pacientes refieren sentirse más compensados de la enfermedad que estos tienen; disminuyen de

1 Ciencia digital, Ambato, Ecuador, luisefrainvelastegui@cienciadigital.org https://orcid.org/0000-0002-7353-0158

${ }^{2}$ Ciencias Médicas Mayabeque, Mayabeque, Cuba, jgarcia@infomed.sld.cu ID https://orcid.org/0000-00027353-0159

${ }^{3}$ Ciencias Médicas Mayabeque, Mayabeque, Cuba, lborges@infomed.sld.cu $1 D$ https://orcid.org/0000-00027353-0160 
sphygmomanometer, stethoscope, digital weight and measuring tape.

Keywords: Hypertension, elderly, physical exercises. este modo sus síntomas propios; utilizando materiales como: esfigmomanómetro, estetoscopio, pesa digital y la cinta métrica.

Palabras claves: Hipertensión arterial, adulto mayor, ejercicios físicos.

\section{Introducción.}

Determinar el comportamiento de la hipertensión arterial en adultos mayores pertenecientes al consultorio No 27 del municipio de Güines antes y después de una intervención terapéutica.

\section{Desarrollo}

\section{Metodologías Empleadas}

La hipertensión arterial es el principal factor de riesgo cardiovascular. Se la conoce como 'la asesina silenciosa", porque en la mayoría de casos no presenta síntomas, por lo que se pueden llegar a desarrollar problemas cardiacos o renales sin ser conscientes de padecer hipertensión. Sin tratamiento, la presión arterial alta aumenta apreciablemente el riesgo de un ataque al corazón o un accidente cerebrovascular.

La hipertensión arterial en el adulto mayor es, en todas sus formas de presentación (sistólica, diastólica, media, sistólica aislada), un factor de riesgo independiente para insuficiencia cardíaca, accidente cerebrovascular, cardiopatía isquémica, insuficiencia renal y, en general, daño de todos los órganos diana. La hipertensión sistólica en este grupo etario es la de mayor prevalencia y fue considerada, hasta hace poco más de tres décadas y por muchos autores, como un mecanismo de adaptación que permitía una correcta presión de perfusión tisular.

Métodos utilizados: pesquisa para identificar los adultos mayores con hipertensión arterial de consultorio No 27 de Güines.

Materiales utilizados: Esfigmomanómetro, estetoscopio, pesa digital, cinta métrica.

Aplicamos a un grupo de pacientes del consultorio No 27 de Güines una batería de de ejercicios de rutina y dieta adecuada en calorías con el fin de controlar las cifras de PA y elevar la calidad de vida de los pacientes hipertensos, en el período comprendido de septiembre del 2019 a noviembre de 2019:

La muestra se conformó con 30 pacientes hipertensos con cifras de tensión arterial entre $140 / 90$ y $180 / 110 \mathrm{mmHg}$ 
A todos los pacientes se le registró edad, sexo, peso, talla e índice de masa corporal (IMC)

Además, se midió la frecuencia cardíaca y la tensión arterial y se repitió la medición después de 3 min de realizar ejercicio físico durante 3 meses.

Se realizó una rutina de ejercicio con la muestra seleccionada ,en la sesión de la tarde en el estadio Héroes del Mayabeque con una duración de 40 min el primer mes y a partir de los otros dos meses de 50 min a 1 hora, todos estos pacientes pertenecen al nivel III (según el Dr. en Ciencias Osmely Prado Sosa)

Nivel III: Adultos Físicamente Independientes: En este grupo se ubican los adultos que realizan todas las actividades de la vida diaria. Ejecutan trabajos físicos leves, cuidan de la casa, realizan las actividades que más les gustan y que exigen poco gasto energético tales como trabajos manuales, caminatas, labores de jardinería, bailes, manejar.

\section{Rutina de ejercicios físicos}

\section{1ra Semana}

\section{Lunes}

- Frecuencia cardíaca en reposo.

- Calentamiento general.

- Calentamiento específico.

- Condición física. 5 minutos.

- Caminata de 20 minutos. Frecuencia cardíaca.

- Estirarse 5 minutos. Frecuencia cardíaca al final de la actividad.

Martes

- Frecuencia cardíaca en reposo.

- Calentamiento general.

- Calentamiento específico.

- Flexibilidad

- Caminata de 25 minutos. Frecuencia cardíaca. 
- Estirarse 5 minutos. Frecuencia cardíaca al final de la actividad.

\section{Miércoles}

- Frecuencia cardíaca en reposo.

- Calentamiento general.

- Calentamiento específico.

- Condición física general8 minutos.

- Caminata de 30 minutos. Frecuencia cardíaca.

- Estirarse 5 minutos. Frecuencia cardíaca al final de la actividad.

Jueves

- Frecuencia cardíaca en reposo.

- Calentamiento general.

- Calentamiento específico.

- Circuito moderado. Brazo, tronco y pierna.

- Frecuencia cardíaca.

- Caminata de 25 minutos. Frecuencia cardíaca.

- Estirarse 5 minutos. Frecuencia cardíaca al final de la actividad.

\section{Viernes}

- Frecuencia cardíaca en reposo.

- Calentamiento general.

- Calentamiento específico y flexibilidad.

- $\quad$ Paseo de 30 a 35 minutos.

- $\quad$ Estirarse 5 minutos.

\section{2da Semana}


Lunes

- Frecuencia cardíaca en reposo.

- Calentamiento general y calentamiento específico.

- Condición física 8 minutos.

- Caminata de 25 minutos.

- Estirarse 5 minutos.

Martes

- Frecuencia cardíaca en reposo.

- Condición general.

- Condición específica.

- Flexibilidad.

- Caminata de 30 minutos.

- Estirarse 5 minutos.

Jueves

- Frecuencia cardíaca en reposo.

- Calentamiento general.

- Calentamiento específico.

- Circuito moderado.

- Caminata de 30 minutos.

- Estirarse 5 minutos.

\section{3ra Semana}

Lunes

- Calentamiento general. 
- Calentamiento específico.

- Condición física general 10 minutos.

- Caminata de 30 minutos.

- Estirarse 5 minutos.

Miércoles

- Calentamiento general.

- Calentamiento específico.

- Condición física general 15 minutos.

- Caminata de 40 minutos.

- $\quad$ Estirarse 5 minutos.

Viernes

- Calentamiento general.

- Calentamiento específico.

- Flexibilidad.

- Caminata de 30 a 35 minutos.

- Estirarse 5 minutos.

\section{4ta semana}

Lunes

- Caminata de 20 minutos. Condición física general 10 minutos.

Martes

- Caminata de 25 minutos. Flexibilidad.

Miércoles

- Caminata de 30 minutos. Condición física general 12 minutos. 


\section{Jueves}

- Caminata de 25 minutos. Circuito.

\section{Viernes}

- Caminata de 30 minutos. Flexibilidad.

A partir del segundo mes se aumenta el tiempo de caminata manteniendo la preparación física en cuanto a las diferencias individuales de los adultos mayores. Se debe lograr que participen en el último mes de $55 \mathrm{~min}$ a 1 hora y 15 min de caminata.

Recomendaciones dietéticas aplicadas a los pacientes con hipertensión arterial

Se recomienda adoptar una alimentación basada en: Frutas, hortalizas y verduras, cereales integrales y derivados: pasta, arroz, pan... Lácteos desnatados o semidesnatados, carnes magras: pollo, conejo, pavo... Pescado azul, frutos secos.

En las siguientes tablas se muestran datos acerca de la evolución de los pacientes de la tercera edad con HTA antes y después de una rutina de ejercicios, así como los resultados alcanzados.

Tabla 1. Edad y sexo

\begin{tabular}{|c|c|c|c|}
\hline \multirow{2}{*}{ Rango de edades } & \multicolumn{2}{|c|}{ Sexo según los rangos de edades } & \multirow{2}{*}{ Total } \\
\cline { 2 - 3 } & Femenino & Masculino & \\
\hline $60-65$ & 7 & 3 & 10 \\
\hline $66-70$ & 3 & 6 & 9 \\
\hline $71-75$ & 4 & 7 & 11 \\
\hline TOTAL & 14 & 16 & 30 \\
\hline
\end{tabular}

Fuente: Elaboración propia.

Tabla 2. Talla

\begin{tabular}{|c|c|}
\hline Rango de talla & $\begin{array}{c}\text { Cantidad de } \\
\text { pacientes }\end{array}$ \\
\hline $1.50-1.55$ & 8 \\
\hline $1.56-1.60$ & 7 \\
\hline $1.61-1.65$ & 9 \\
\hline $1.66-1.70$ & 4 \\
\hline $1.71-1.75$ & 0 \\
\hline
\end{tabular}




\begin{tabular}{|c|c|}
$1.76-1.80$ & 2 \\
\hline TOTAL & $\mathbf{3 0}$ \\
\hline
\end{tabular}

Fuente: Elaboración propia.

Tabla 3. Peso corporal antes de la rutina de ejercicio y después de la rutina de ejercicio.

\begin{tabular}{|c|c|c|}
\hline Rango de peso & $\begin{array}{c}\text { Cantidad de } \\
\text { pacientes antes }\end{array}$ & $\begin{array}{c}\text { Cantidad de } \\
\text { pacientes después }\end{array}$ \\
\hline $60-70$ & 22 & 26 \\
\hline $71-80$ & 7 & 4 \\
\hline $81-90$ & 0 & 0 \\
\hline $91-100$ & 1 & 0 \\
\hline TOTAL & $\mathbf{3 0}$ & $\mathbf{3 0}$ \\
\hline
\end{tabular}

Fuente: Elaboración propia.

En la tabla No 3 se muestran mejorías, puesto que los pacientes que estaban en el rango de 81-90 y de 91-100 disminuyeron su peso corporal, al igual que los demás pacientes disminuyendo aproximadamente $7 \mathrm{~kg}$.

Tabla 4. Índice de masa corporal (IMC) antes de la rutina de ejercicios, y después de la rutina de ejercicios.

\begin{tabular}{|c|c|c|c|c|}
\hline \multirow[b]{2}{*}{ IMC } & \multicolumn{2}{|c|}{ Antes } & \multicolumn{2}{|c|}{ Después } \\
\hline & $\begin{array}{c}\text { No. de } \\
\text { pacientes }\end{array}$ & $\%$ & $\begin{array}{c}\text { No. de } \\
\text { pacientes }\end{array}$ & $\%$ \\
\hline $20-25$ & 3 & 10 & 11 & 36.7 \\
\hline $26-30$ & 27 & 90 & 19 & 63.3 \\
\hline TOTAL & 30 & 100 & 30 & 100 \\
\hline
\end{tabular}

Fuente: Elaboración propia.

En la tabla No 4 se muestran mejorías ya que se logró reducir el número de pacientes con sobrepeso e incluso algunos pacientes alcanzaron su peso ideal. 
Tabla 5. Presión arterial antes de la rutina de ejercicio y después de la rutina de ejercicio

\begin{tabular}{|c|c|c|}
\hline Presión arterial & $\begin{array}{c}\text { Cantidad de } \\
\text { pacientes antes }\end{array}$ & $\begin{array}{c}\text { Cantidad de } \\
\text { pacientes después }\end{array}$ \\
\hline $140 / 90-140 / 110$ & 8 & 21 \\
\hline $150 / 90-150 / 110$ & 12 & 8 \\
\hline $160 / 90-160 / 110$ & 8 & 1 \\
\hline $170 / 90-170 / 110$ & 1 & 0 \\
\hline $180 / 90-180 / 110$ & 1 & 0 \\
\hline TOTAL & 30 & 30 \\
\hline
\end{tabular}

Fuente: Elaboración propia.

En la tabla No5 se muestran mejorías ya que se logró estabilizar la presión de todos los pacientes, principalmente los que se encontraban descompensados obteniendo presiones favorables entre 140/90 y 150/110 en la mayoría de los casos.

\section{Conclusiones.}

- El plan de ejercicios propicia una herramienta de trabajo como alternativa para disminuir los índices de hipertensión arterial en los adultos mayores. La dieta rica en frutas, vegetales, carnes blancas, frutos secos, cereales, contribuyen a la estabilidad de la hipertensión arterial en pacientes hipertensos. Encontrándose tanto el ejercicio físico como la dieta en una estrecha relación puesto que ambos estabilizan la salud de los pacientes.

\section{Referencias bibliográficas.}

Jorge Cevallos Díaz. (2017). El Adulto Mayor y la actividad física. Material digital.

Alfredo Dueñas Herrera (2015). Hipertensión Arterial Guía para la prevención,diagnóstico y tratamiento. La Habana, Editorial Ciencias Médicas.

Dr.COsmery Prado Sosa (2016). Programa de Actividades Físicas para el tratamiento de la Hipertensión. La Habana.

Fernando Villavicencio Porro. (2015). Psicología y Salud. La Habana, Editorial Ciencias Médicas.

Oliveros J (2009).La HTA en el adulto mayor. Universidad de Madrid (inédito) 
PARA CITAR EL ARTÍCULO INDEXADO.

Velasteguí López, E., García Espinosa, J., \& Borges Sierra, L. (2019). Intervención educativa sobre hipertensión arterial en la tercera edad del Consultorio No 27 de Güines. Anatomía Digital, 2(1), 57-66. https://doi.org/10.33262/anatomiadigital.v2i1.1124

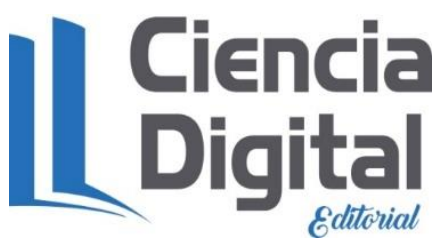

El artículo que se publica es de exclusiva responsabilidad de los autores y no necesariamente reflejan el pensamiento de la Revista Anatomía Digital.

El artículo queda en propiedad de la revista y, por tanto, su publicación parcial y/o total en otro medio tiene que ser autorizado por el director de la Revista Anatomía Digital.
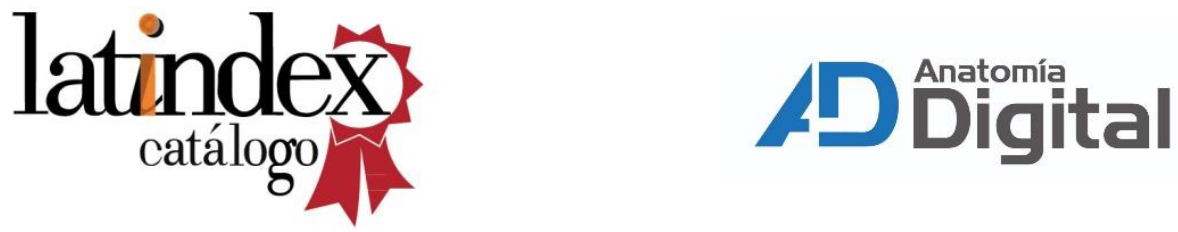